\title{
The orbit of Phoebe from Earthbased and Voyager observations
}

\author{
R.A. Jacobson \\ 301-150 Jet Propulsion Laboratory, California Institute of Technology, 4800 Oak Grove Drive, Pasadena, CA 91109-8099, U.S.A.
}

Received September 17, 1996; accepted June 16, 1997

\begin{abstract}
This article presents the results of a fit of a numerically integrated orbit for the Saturnian satellite Phoebe to Earthbased astrometric observations (from 1904 to 1996) and imaging data acquired by the Voyager 2 spacecraft during its encounter with Saturn. The primary results are the epoch state vector for the integration and a set of mean orbital elements which approximately represent the orbit. An assessment of the quality of the fit and the accuracy of the orbit is also provided.
\end{abstract}

Key words: ephemerides — planets and satellites: general — planets and satellites: individual: phoebe

\section{Introduction}

The ninth satellite of Saturn, Phoebe, was discovered by W.H. Pickering in March 1899 from photographs made in 1898 at the Harvard observatory station, Arequipa, Peru (Pickering 1899). He subsequently published preliminary ephemerides for the satellite (Pickering 1905a,b). Ross (1905) produced the first definitive orbit based on observations made at Arequipa, Yerkes, and Lick in the early 1900s; Zadunaisky (1954) updated Ross' orbit using observations made between 1907 and 1942. Rose (1979) generated the first numerically integrated Phoebe orbit which he fit to observations over the period 1904 to 1969 ; he omitted those from Arequipa. Later integrations were done by Bec-Borsenberger \& Rocher (1982) who fit observations from 1904 to 1981 and by Bykova \& Shikhalev (1982, 1984) who fit observations from 1898 to 1981.

The purpose of this work is to update the orbit in light of recent astrometric observations and imaging observations obtained by the Voyager 2 spacecraft. Further incentive is provided by NASA's Cassini mission to Saturn

Send offprint requests to: R.A. Jacobson
(Kohlhase 1993). Current plans call for the Cassini spacecraft to pass by Phoebe at a distance of about $50000 \mathrm{~km}$. A high quality ephemeris is needed to support scientific observations during this flyby.

\section{Orbit model}

Our model for Phoebe's orbit is a numerical integration of its equation of motion (Peters 1981) which includes the effects of an oblate Saturn, perturbations from seven of the eight major Saturnian satellites (Hyperion, whose mass is unknown but presumed quite small, is neglected), and perturbations from the Sun, Jupiter, and Uranus. The formulation is in Cartesian coordinates centered at the Saturnian system barycenter and referenced to the Earth mean equator and equinox of J2000 system. Because it is small and its mass unknown, Phoebe, like Hyperion, is assumed to be massless, hence the barycenter location depends only upon the planet and perturbing satellites. JPL planetary ephemeris DE403 (Standish et al. 1995) provides the masses and positions of the Sun and perturbing planets, and JPL satellite ephemeris SAT077 (Jacobson 1996a) provides those of the perturbing satellites.

The model is far more complete than necessary to fit the observations. Rose's original work included only solar perturbations, that of Bykova and Shikhalev accounted for the Sun and Jupiter, and that of Bec-Borsenberger and Rocher took into account the effects of Titan, Jupiter, and the Sun. All three of these previous investigations obtained reasonable fits to the observations. We selected the model in this work primarily for consistency with the integrations of the other Saturnian satellites being performed in preparation for support of the Cassini mission (Jacobson 1996b). Table 1 gives the ratio of the maximum perturbing accelerations encountered in our integrations to the average point mass central body acceleration. The table entries show that Phoebe's acceleration due to Jupiter is about $0.47 \%$ that of the Sun and due to Titan is about $0.13 \%$. Interestingly, the effect of Uranus is greater than that of any of the remaining major satellites. We also investigated a simplified model which replaces the 
perturbing satellites, except for Titan, with uniform circular equatorial rings retaining only their quadrupole effect (see Roy et al. 1988 for a discussion of this technique). In addition, the simplified model obtains its Titan positions from a precessing ellipse approximate representation of the Titan ephemeris (the elements for the ellipse can be found in Appendix A). Integrations with the simplified model differ from those with the complete model by less than $50 \mathrm{~km}$ over a 50 year period. In fact, because such differences are well below the accuracy of the pre1966 observations (the integration epoch is November 11, 1966), we processed those early observations with only the simplified model.

Table 1. Ratio of the maximum perturbing acceleration to the central body acceleration

\begin{tabular}{llll}
\hline Accel. & Magnitude & Accel. & Magnitude \\
\hline Sun & $3.2 \quad 10^{-1}$ & Dione & $2.910^{-6}$ \\
Jupiter & $1.510^{-3}$ & Tethys & $1.610^{-6}$ \\
Titan & $4.110^{-4}$ & Harmonics & $1.010^{-6}$ \\
Uranus & $1.310^{-5}$ & Enceladus & $1.910^{-7}$ \\
Iapetus & $8.510^{-6}$ & Mimas & $9.710^{-8}$ \\
Rhea & $6.310^{-6}$ & & \\
\hline
\end{tabular}

The integration was carried out with a variable step size, variable order, Gauss-Jackson method. An absolute truncation error limit of $10^{-10} \mathrm{~km} \mathrm{~s}^{-1}$ imposed on the velocity controlled the integration step. The average step size was 25896 seconds and the maximum order was 15 .

\section{Observations}

\subsection{Sources and summary}

The literature search of Pierce (1975) lists publications containing micrometric and photographic Phoebe observations over the period 1898 to 1969 . Bec-Borsenberger \& Rocher (1982) extend the list to include photographic observations from 1975 to 1981. In addition, they provide several photographic observations made at La Silla in 1981 which apparently have not been published elsewhere. The only observations published subsequent to 1981 appear to be those made at La Silla in 1982 (Debehogne 1984). The U.S. Naval Observatory (Rohde 1994) and McDonald Observatory (Whipple 1992, 1995) supplied recent unpublished photographic observations, and McDonald Observatory (Whipple 1996) supplied unpublished CCD observations. Requests for the unpublished USNO and McDonald observations must be made to the respective observers. During its encounter with Saturn in 1981, the Voyager 2 spacecraft acquired eight images of Phoebe against a star background. Appendix B provides position measures derived from these images.
Table 2 summarizes of all of the currently available Earthbased observations grouped into sets. The first column contains the year of the observations; the second and third columns identify the observatory and instrument. A reference or publication is given in column four (see the References section of this paper). Finally, the code number in the last column uniquely identifies the observation set.

\subsection{Comments}

The observations in Set 29 made at the Crimean Astrophysical Observatory are reported together with observations of Hyperion and Iapetus. However, the differential positions of Phoebe relative to those bodies exhibit significant errors in right ascension. For this reason, we preferred the absolute rather than differential positions.

The McDonald observations by Mulholland and Shelus, the Lowell observations by Bowell, and the La Silla observations by Debehogne all include a number of simultaneous observations of other satellites. For these sets we constructed and used satellite-relative rather than the absolute positions.

Rohde's USNO set 37 has a significant bias in right ascension, and his set 39 has a significant bias in declination. Until the sources of these biases are understood, the observations are not usable.

The Voyager observations are the pixel and line locations of the images of Phoebe and background reference stars in the Voyager camera frame. They provide measures of Phoebe's position with accuracies in the 70 to $260 \mathrm{~km}$ range. The FK4/B1950 positions of the stars are from a special star catalogue (Klemola et al. 1979) made for the Voyager project and reduced to the Perth 70 catalogue. To facilitate processing of the observations, we rotated the star positions to the FK5/J2000 system following the IAU Commission 20 conversion procedure (Standish et al. 1992).

\section{Orbit determination}

\subsection{Observation modeling}

The determination of the orbit employs an algorithm which minimizes the sum of squares of the observedminus-computed observation residuals. This procedure requires the formation of computed observables. In our approach, we attempt, where possible, to compute the values of the observables as actually reported rather than transform those values to a standard system (e.g. B1950 or J2000 system) as other authors have done.

Because the integration is performed using the DE403 planetary ephemeris and SAT077 satellite ephemeris for perturbing body positions, the Phoebe orbit is in fact generated in the J2000 reference frame of the International Earth Rotation Service (IERS). The observation processing software also refers the Earth orientation (observer 
Table 2. Earthbased astrometric observation sets

\begin{tabular}{|c|c|c|c|c|}
\hline Year & Observatory & Instrument & Reference & Code \\
\hline 1898 & Arequipa & 24 in Bruce & Pickering (1908) & 1 \\
\hline 1899 & Arequipa & 24 in Bruce & Pickering (1908) & 2 \\
\hline 1900 & Arequipa & 24 in Bruce & Pickering (1908) & 3 \\
\hline 1902 & Arequipa & 24 in Bruce & Pickering (1908) & 4 \\
\hline 1904 & Arequipa & 24 in Bruce & Pickering (1905a,1905b) & 5 \\
\hline 1904 & Yerkes & 40 in refactor & Barnard (1905) & 6 \\
\hline 1904 & Lick & Crossley reflector & Perrine (1904) & 7 \\
\hline 1905 & Arequipa & 24 in Bruce & Pickering (1906a) & 8 \\
\hline 1905 & Lick & Crossley reflector & Albrecht \& Smith (1909) & 9 \\
\hline 1906 & Lick & Crossley reflector & Perrine (1909) & 10 \\
\hline 1906 & Arequipa & 24 in Bruce & Pickering (1906b) & 11 \\
\hline 1906 & Arequipa & 24 in Bruce & Pickering (1906c) & 12 \\
\hline 1906 & Yerkes & 40 in refactor & Barnard (1906) & 13 \\
\hline 1907 & Greenwich & 30 in reflector & Christie (1909) & 14 \\
\hline 1908 & Lick & Crossley reflector & Perrine (1909) & 15 \\
\hline 1908 & Greenwich & 30 in reflector & Christie (1910) & 16 \\
\hline 1909 & Greenwich & 30 in reflector & Christie (1911) & 17 \\
\hline 1910 & Greenwich & 30 in reflector & Christie (1912) & 18 \\
\hline $1912 / 13$ & Yerkes & 40 in refactor & Barnard (1913) & 19 \\
\hline 1913 & Yerkes & 40 in refactor & Barnard (1914) & 20 \\
\hline 1922 & Yerkes & 24 in reflector & van Biesbroeck (1922) & 21 \\
\hline 1940 & Mt. Wilson & 100 in reflector & Nicholson \& Richmond (1944) & 22 \\
\hline 1942 & McDonald & 82 in reflector & van Biesbroeck (1944) & 23 \\
\hline 1952 & Cordoba & Normal astrograph & Bobone (1953) & 24 \\
\hline 1955 & Yerkes & 24 in reflector & van Biesbroeck (1956) & 25 \\
\hline 1955 & McDonald & 82 in reflector & van Biesbroeck (1956) & 26 \\
\hline 1957 & Bloemfontien & ADH telescope & van Biesbroeck (1958) & 27 \\
\hline 1960 & Flagstaff & 40 in reflector & Roemer \& Lloyd (1966) & 28 \\
\hline 1968 & Crimean & $40 \mathrm{~cm}$ astrograph & Chernykh \& Chernykh (1971) & 29 \\
\hline 1969 & Kitt Peak & $213 \mathrm{~cm}$ reflector & van Biesbroeck et al. (1976) & 30 \\
\hline 1969 & Catalina & $154 \mathrm{~cm}$ reflector & van Biesbroeck et al. (1976) & 31 \\
\hline $1975 / 76$ & McDonald & $2.1 \mathrm{~m}$ reflector & Mulholland \& Shelus (1980) & 32 \\
\hline 1981 & Lowell, Mesa stn. & $0.33 \mathrm{~m}$ reflector & Bowell (1981/82) & 33 \\
\hline 1981 & La Silla & $40 \mathrm{~cm}$ GPO & Debehogne (1981a-d/82) & 34 \\
\hline 1982 & Lowell, Mesa stn. & $0.33 \mathrm{~m}$ reflector & Bowell (1988) & 35 \\
\hline 1982 & La Silla & $40 \mathrm{~cm} \mathrm{GPO}$ & Debehogne (1984) & 36 \\
\hline 1992 & USNO & 24 in reflector & Rohde (1994) & 37 \\
\hline 1992 & McDonald & $2.1 \mathrm{~m}$ reflector & Whipple (1992) & 38 \\
\hline 1993 & USNO & 24 in reflector & Rohde (1994) & 39 \\
\hline 1994 & McDonald & $2.1 \mathrm{~m}$ reflector & Whipple (1995) & 40 \\
\hline $1995 / 96$ & McDonald & $2.1 \mathrm{~m}$ reflector & Whipple (1996) & 41 \\
\hline
\end{tabular}

location) to that same frame. The first step in computing the observables is the calculation of the natural $d i$ rection from the observer to Phoebe in the IERS/J2000 system (see Murray 1983). For observations referred to the FK5/J2000 system, we form computed observables directly from the IERS/J2000 natural direction; the frame tie between the two systems is presumed to be much better than the error in the star catalogues used in the reduction of the observations. For observations referred to a mean equator of epoch system (this includes the FK4/B1950 system), we first precess the IERS/J2000 natural direction to the mean equator at the time of the observation with the IAU76 precession, we then precess it from that time to the mean equator of epoch with the Newcomb precession. In addition, we apply corrections for the FK4-FK5 equinox offset and the elliptic aberration. With the exception of those from Yerkes, all observations are available in a mean equator system.

The Yerkes observations, published as apparent positions, were originally reduced with the aid of star catalogues (i.e., catalogue mean places of stars). Before processing, we converted these apparent positions back to 
mean-of-date positions by correcting for the circular part of the stellar aberration and for the nutation. As we have found no record of the constants of aberration used by the observers to construct their apparent positions, we used a value of $20^{\prime \prime} 47$ (Paris conference 1911). The E.W. Woolard theory (Explanatory Supplement 1961) provided the nutation corrections rather than the 1980 IAU theory currently in use.

We should also comment that as an experiment we computed the observables corresponding to Yerkes observations directly as apparent positions in the J2000 system (see the 1987 Astronomical Almanac for the procedure). The residuals for these computed apparent positions against the published apparent positions were not significantly different from those obtained for the computed mean-of-date positions against the observations corrected to mean-of-date positions.

The Voyager observations are modelled in JPL's Optical Navigation Program (Owen \& Vaughan 1991). This software package computes the pixel and line locations of the Phoebe and star images using a model of the Voyager camera, the trajectory of the Voyager spacecraft, the Phoebe orbit, and the catalogue positions of the stars. The spacecraft trajectory was the same one used in the SAT077 major satellite ephemeris development.

\subsection{Observation processing}

For each observation set, we used Householder transformations (Lawson \& Hanson 1974) to pack the matrix of weighted observation partial derivatives and the weighted residual vector into an upper triangular square root information matrix and associated residual vector. This matrix and vector constitute the square root information array which is equivalent to the normal equations. Each column of the matrix and each element of the vector are associated with an epoch state vector component. Combining the separate information arrays via Householder transformations led to the square root information array for the complete data set. The solution for the state vector was generated and analyzed by means of singular value decomposition techniques (Lawson \& Hanson 1974) applied to the composite square root information array.

The weight assigned to each observation set is based on our assessment of the quality of the data in the set; numerically it is the reciprocal of our assumed accuracy or standard error for the set. After discussions with the observer, we selected accuracies of 0 ".5 for the 1992 and 1994 photographic McDonald observations and 0".4 for the 1996 CCD observations. The remainder of the accuracies we determined through an iterative procedure in which we picked a set of weights, fit the observations, computed a new orbit, and examined the statistics of the residuals associated with that orbit. In general, for a particular observation set we took the accuracy to be equal to the root-mean-square of the residuals. However, we also im- posed a lower limit of 0.5 on the accuracy of the absolute positions. This limit follows from our assumption that the recent McDonald observations are representative of the best photographic absolute positions normally expected for a distant dim object such as Phoebe. The limit mainly affects data sets with only one or two observations. For the relative observations we imposed a lower limit of $0^{\prime \prime} .2$, a value typically associated with photographic relative observations of the Saturnian satellites. Most observation sets actually have two separate weights assigned: one for right ascension, $\alpha$, or relative right ascension, $\Delta \alpha$, and the other for declination, $\delta$, or relative declination, $\Delta \delta$. The weights for the image locations in the Voyager data correspond to accuracies of 0.5 pixel; the same weights were used during Voyager operations.

The publications of the Greenwich observations give positions of Saturn which were reduced in the same manner as those of Phoebe. In the publications these positions are compared to the tabular place of Saturn in the Nautical Almanac in order to develop a set of corrections for the elimination of star catalog and Saturn ephemeris errors. Rather than apply the published corrections, we chose instead to determine our own corrections through direct processing of the Saturn positions. We formed residuals against positions computed from the DE403 and SAT077 ephemerides (the former gives the Saturnian system barycenter position and the latter relates the planet position to the barycenter). We then extended our processing to include the determination of right ascension and declination biases which minimize these residuals. The biases affect both the Phoebe and Saturn positions. The accuracy for the Saturn positions was set at 0.5 .

\section{Processing results}

\subsection{Observation residuals}

Table 3 gives the postfit statistics for the observedminus-computed residuals for the Earthbased observations grouped by data set. For each set the table indicates the type of observations and the number used versus the total number available. The statistics include the sample mean $(\mu)$ of the residuals, the standard deviation $(\sigma)$ about the mean, and the root-mean-square (rms). The Arequipa, the 1904 Yerkes, and the USNO observations were not fit; however, statistics for them are included. To arrive at the statistics for the unused observations, we deleted all residuals greater than $30^{\prime \prime}$ and applied a $3 \sigma$ rejection criterion to the remainder.

The mean for each set gives an indication of systematic errors. For example, a large mean in the right ascension residuals could be attributed to an equinox offset. The standard deviation measures the scatter of the residuals about the mean and characterizes the noise level of the observations, and the root-mean-square represents the overall quality of the fit. Also included is the weighted 
Table 3. Astrometric observation residual statistics. The column entitled "Type" gives the observation type: the letter $\mathrm{V}$ denotes visual, $\mathrm{P}$ denotes photographic, and $\mathrm{C}$ denotes $\mathrm{CCD}$. The digit gives measurement type: $0-$ absolute $\alpha$ and $\delta ; 1-$ $\Delta \alpha$ and $\Delta \delta ; 2$ - angular separation and $\Delta \delta$. The column entitled "No." gives the number of observations included in the solution/number of available observations, $\mu$ denotes the sample mean, $\sigma$ denotes the standard deviation about the mean, rms denotes the root-mean-square, and wrms denotes the root-mean-square of the weighted residuals

\begin{tabular}{|c|c|c|c|c|c|c|c|c|c|c|c|c|c|}
\hline Set & Year & Observer/Source & Type & No. & $\mu$ & $\sigma$ & $\mathrm{rms}$ & wrms & No. & $\mu$ & $\sigma$ & $\mathrm{rms}$ & wrms \\
\hline 1 & 1898 & Pickering & $\mathrm{P} 0$ & $7 / 7$ & $-0^{\prime \prime} \cdot 29$ & $4^{\prime \prime} .16$ & $3^{\prime \prime} 86$ & & $7 / 7$ & $2^{\prime \prime} 63$ & $3^{\prime \prime} \cdot 76$ & $4^{\prime \prime} \cdot 36$ & \\
\hline 2 & 1899 & Pickering & P0 & $0 / 3$ & & & & & $0 / 3$ & & & & \\
\hline 3 & 1900 & Pickering & P0 & $20 / 26$ & $8^{\prime \prime} .31$ & $6^{\prime \prime} \cdot 24$ & $10^{\prime \prime} \cdot 3$ & & $20 / 26$ & $3^{\prime \prime} \cdot 16$ & $5 ! .87$ & $6^{\prime \prime} .53$ & \\
\hline 4 & 1902 & Pickering & P0 & $2 / 6$ & $-11^{\prime \prime} 4$ & $19 ! 8$ & $18^{\prime \prime} \cdot 0$ & & $2 / 6$ & $-3^{\prime \prime} 15$ & $0 ! 31$ & $3^{\prime \prime} 16$ & \\
\hline 5 & 1904 & Pickering & $\mathrm{P} 2$ & $26 / 28$ & $6^{\prime \prime} .55$ & $4^{\prime \prime} .55$ & $7^{\prime \prime} \cdot 92$ & & $26 / 28$ & $-3^{\prime \prime} 68$ & $7^{\prime \prime} \cdot 42$ & $8^{\prime \prime} .15$ & \\
\hline 6 & 1904 & Barnard & V0 & $1 / 2$ & $10^{\prime \prime} .3$ & $0^{\prime} .00$ & $10^{\prime \prime} \cdot 3$ & & $1 / 2$ & $-3^{\prime \prime} .90$ & $0 ! 00$ & $3^{\prime \prime} \cdot 90$ & \\
\hline 7 & 1904 & Perrine & P0 & $5 / 6$ & $0 ! 47$ & $0 ! 99$ & $1^{\prime \prime} .01$ & 1.01 & $5 / 6$ & $-0^{\prime} ! 01$ & $0 ! 58$ & $0 ! 52$ & 0.95 \\
\hline 8 & 1905 & Pickering & P2 & $11 / 17$ & $-2^{\prime \prime} 43$ & $12^{\prime \prime} .1$ & $11^{\prime \prime} 8$ & & $11 / 17$ & $-4^{\prime \prime} .49$ & $14^{\prime \prime} .2$ & $14^{\prime \prime} .2$ & \\
\hline 9 & 1905 & Albrecht & $\mathrm{P} 0$ & $11 / 11$ & $-1^{\prime \prime} .03$ & $0 ! 94$ & $1^{\prime \prime} \cdot 36$ & 1.01 & $11 / 11$ & $1^{\prime \prime} .52$ & $1^{\prime \prime} .72$ & $2^{\prime \prime} \cdot 24$ & 0.99 \\
\hline 10 & 1906 & Perrine & P0 & $8 / 8$ & $0^{\prime} .08$ & $1^{\prime \prime} .12$ & $1^{\prime \prime} .05$ & 0.96 & $8 / 8$ & $0 ! 54$ & $0 ! 82$ & $0^{\prime \prime} \cdot 94$ & 0.94 \\
\hline 11 & 1906 & Pickering & $\mathrm{P} 2$ & $6 / 6$ & $4^{\prime \prime} .06$ & $3^{\prime \prime} \cdot 70$ & $5^{\prime \prime} \cdot 28$ & & $6 / 6$ & $-5^{\prime \prime} \cdot 26$ & $9 ! 71$ & $10^{\prime \prime} \cdot 3$ & \\
\hline 12 & 1906 & Pickering & $\mathrm{P} 2$ & $9 / 9$ & $-1^{\prime \prime} \cdot 93$ & $8^{\prime \prime} .05$ & $7^{\prime \prime} .83$ & & $9 / 9$ & $-3^{\prime \prime} 45$ & $5^{\prime \prime} \cdot 71$ & $6^{\prime \prime} \cdot 39$ & \\
\hline 13 & 1906 & Barnard & V0 & $11 / 12$ & $-2^{\prime \prime} \cdot 26$ & $4^{\prime \prime} \cdot 25$ & $4^{\prime \prime} .64$ & 1.01 & $12 / 12$ & $-0^{\prime \prime} 63$ & $1^{\prime \prime} .52$ & $1^{\prime \prime} .58$ & 0.99 \\
\hline 14 & 1907 & Christie & P0 & $16 / 16$ & $0 ! 20$ & $0 ! 70$ & $0 ! 71$ & 1.01 & $16 / 16$ & $0 ! 18$ & $0 ! 77$ & $0 ! 76$ & 0.96 \\
\hline 15 & 1908 & Perrine & P0 & $2 / 2$ & $1^{\prime \prime} .91$ & $0^{\prime \prime} 73$ & $1^{\prime \prime} .98$ & 0.99 & $2 / 2$ & $-0^{\prime \prime} 80$ & $0^{\prime} .03$ & $0^{\prime} ! 80$ & 1.01 \\
\hline 16 & 1908 & Christie & P0 & $22 / 23$ & $0^{\prime} \cdot 20$ & $0^{\prime} 63$ & $0^{\prime} \cdot 65$ & 0.99 & $22 / 23$ & $0 ! 04$ & $0 ! \cdot 72$ & $0^{\prime \prime} 71$ & 1.01 \\
\hline 17 & 1909 & Christie & P0 & $12 / 12$ & $0 ! 30$ & $1^{\prime \prime} .07$ & $1^{\prime \prime} .07$ & 1.02 & $12 / 12$ & $-0^{\prime \prime} 04$ & $0 ! 98$ & $0^{\prime \prime} \cdot 94$ & 0.99 \\
\hline 18 & 1910 & Christie & P0 & $7 / 7$ & $-0^{\prime \prime} .01$ & $1^{\prime \prime} .40$ & $1^{\prime \prime} \cdot 29$ & 0.99 & $7 / 7$ & $0^{\prime} ! 40$ & $0 ! 68$ & $0^{\prime \prime} 75$ & 1.00 \\
\hline 19 & 1912 & Barnard & V0 & $12 / 12$ & $1^{\prime \prime} 68$ & $1^{\prime \prime} \cdot 20$ & $2^{\prime \prime} 04$ & 1.02 & $7 / 7$ & $-0^{\prime} \cdot 63$ & $1^{\prime \prime} \cdot 24$ & $1^{\prime \prime} \cdot 30$ & 1.00 \\
\hline 20 & 1913 & Barnard & V0 & $5 / 5$ & $0^{\prime \prime} 84$ & $0^{\prime} ! 66$ & $1^{\prime \prime} .02$ & 0.93 & $3 / 3$ & $-1^{\prime \prime} .08$ & $0^{\prime \prime} 32$ & $1^{\prime \prime} .11$ & 1.01 \\
\hline 21 & 1922 & VanBiesbroeck & P0 & $4 / 5$ & $3^{\prime \prime} 41$ & $0 ! 72$ & $3 ! 47$ & 0.99 & $4 / 5$ & $-1^{\prime \prime} .45$ & $1^{\prime \prime} .26$ & $1^{\prime \prime} .82$ & 0.98 \\
\hline 22 & 1940 & Nicholson & P0 & $1 / 1$ & $-0^{\prime \prime} \cdot 24$ & $0^{\prime} .00$ & $0^{\prime \prime} \cdot 24$ & 0.49 & $1 / 1$ & $0 ! 00$ & $0 ! 00$ & $0^{\prime} .00$ & 0.01 \\
\hline 23 & 1942 & VanBiesbroeck & P0 & $7 / 8$ & $1^{\prime \prime} .66$ & $0 ! 41$ & $1^{\prime \prime} .70$ & 0.97 & $7 / 8$ & $-0^{\prime \prime} \cdot 24$ & $0 ! 25$ & $0^{\prime} \cdot 34$ & 0.67 \\
\hline 24 & 1952 & Bobone & P0 & $7 / 7$ & $-0^{\prime \prime} 02$ & $1^{\prime \prime} .02$ & $0^{\prime \prime} \cdot 95$ & 1.00 & $7 / 7$ & $0 ! 02$ & $0^{\prime} \cdot 33$ & $0^{\prime} ! 31$ & 0.61 \\
\hline 25 & 1955 & VanBiesbroeck & P0 & $8 / 11$ & $1^{\prime \prime} .09$ & 0 '.73 & $1^{\prime \prime} .29$ & 0.96 & $8 / 11$ & $0 ! 45$ & $0^{\prime \prime} 48$ & $0^{\prime} 63$ & 0.98 \\
\hline 26 & 1955 & VanBiesbroeck & P0 & $3 / 3$ & $2^{\prime \prime} .13$ & $0 ! 56$ & $2^{\prime \prime} 18$ & 0.97 & $3 / 3$ & $0 ! 79$ & $0 ! 88$ & $1^{\prime \prime} .07$ & 1.02 \\
\hline 27 & 1957 & VanBiesbroeck & P0 & $8 / 8$ & $0^{\prime \prime} 34$ & $0^{\prime} 69$ & $0^{\prime \prime} 73$ & 0.97 & $8 / 8$ & $1^{\prime \prime} .26$ & $0 ! 70$ & $1^{\prime \prime} .42$ & 0.98 \\
\hline 28 & 1960 & Roemer & P0 & $2 / 2$ & $0 ! 90$ & $0 ! 16$ & $0^{\prime} \cdot 91$ & 1.01 & $2 / 2$ & $0 ! 40$ & $0 ! 17$ & $0 ! 42$ & 0.84 \\
\hline 29 & 1968 & Chernykh & P0 & $2 / 2$ & $0 ! 73$ & $1^{\prime \prime} .91$ & $1^{\prime \prime} .53$ & 0.99 & $2 / 2$ & $-1^{\prime \prime} 16$ & $0 ! 51$ & $1^{\prime \prime} .22$ & 0.97 \\
\hline 30 & 1969 & VanBiesbroeck & P0 & $1 / 3$ & $1^{\prime \prime} .52$ & $0^{\prime} 00$ & $1^{\prime \prime} .52$ & 0.98 & $1 / 3$ & $0^{\prime \prime} 38$ & 0.00 & $0^{\prime} \cdot 38$ & 0.77 \\
\hline 31 & 1969 & VanBiesbroeck & P0 & $1 / 1$ & $-0^{\prime \prime} 13$ & $0^{\prime} \cdot 00$ & $0^{\prime \prime} 13$ & 0.26 & $1 / 1$ & $0 ! 85$ & $0^{\prime} .00$ & $0^{\prime \prime} 85$ & 1.00 \\
\hline \multirow[t]{2}{*}{32} & 1975 & Mulholland & $\mathrm{P} 1$ & $4 / 5$ & $-0^{\prime \prime} \cdot 26$ & $0^{\prime} ! 27$ & $0^{\prime} \cdot 35$ & 0.99 & $4 / 5$ & $-0^{\prime \prime} \cdot 06$ & $0^{\prime} .07$ & 0 '.09 & 0.45 \\
\hline & & & P0 & $2 / 3$ & $-0^{\prime \prime} \cdot 28$ & $0^{\prime \prime} 81$ & $0^{\prime} ! 64$ & 0.98 & $2 / 3$ & $-0^{\prime \prime} 16$ & $0 ! 19$ & $0^{\prime} \cdot 21$ & 0.42 \\
\hline \multirow[t]{2}{*}{33} & 1981 & Bowell & $\mathrm{P} 1$ & $8 / 10$ & $1^{\prime \prime} .08$ & $0^{\prime} ! 66$ & $1^{\prime \prime} \cdot 24$ & 0.99 & $8 / 10$ & $-0^{\prime \prime} .57$ & $0 ! 85$ & $0^{\prime \prime} 98$ & 0.98 \\
\hline & & & P0 & $2 / 2$ & $-0^{\prime} ! 41$ & $0 \prime 57$ & 0.58 & 0.96 & $2 / 2$ & $-1^{\prime \prime} .03$ & $0^{\prime} \cdot 20$ & $1^{\prime \prime} .04$ & 0.99 \\
\hline 34 & 1981 & Debehogne & $\mathrm{P} 1$ & $20 / 21$ & $0 ! 44$ & $0 ! 43$ & $0^{\prime} \cdot 61$ & 0.94 & $20 / 21$ & $0 ! 00$ & $0 ! 58$ & $0 ! 57$ & 0.95 \\
\hline 35 & 1982 & Bowell & $\mathrm{P} 1$ & $2 / 2$ & $-0^{\prime \prime} \cdot 29$ & $0^{\prime} ! 63$ & $0^{\prime \prime} .53$ & 0.96 & $2 / 2$ & $-0^{\prime \prime} \cdot 95$ & $1^{\prime \prime} .14$ & $1^{\prime \prime} .25$ & 1.00 \\
\hline 36 & 1982 & Debehogne & $\mathrm{P} 1$ & $18 / 21$ & $-0^{\prime \prime} 34$ & $0^{\prime \prime} 48$ & $0^{\prime \prime} \cdot 58$ & 0.96 & $18 / 21$ & $-0^{\prime} \cdot 08$ & $0^{\prime} 60$ & $0^{\prime \prime} 59$ & 0.98 \\
\hline 37 & 1992 & Rohde & P0 & $22 / 22$ & $1^{\prime \prime} .89$ & $0^{\prime \prime} 36$ & $1^{\prime \prime} .93$ & & $22 / 22$ & $-0^{\prime} ! 26$ & $0^{\prime} \cdot 23$ & $0^{\prime \prime} \cdot 34$ & \\
\hline 38 & 1992 & Whipple & P0 & $12 / 12$ & $-0^{\prime \prime} 10$ & $0^{\prime \prime} \cdot 25$ & $0^{\prime \prime} \cdot 26$ & 0.52 & $12 / 12$ & $-0^{\prime \prime} .06$ & $0 ! 50$ & $0^{\prime \prime} 49$ & 0.97 \\
\hline 39 & 1993 & Rohde & P0 & $9 / 9$ & $0^{\prime} ! 15$ & $0^{\prime \prime} 26$ & $0^{\prime} \cdot 28$ & & $9 / 9$ & $-1^{\prime \prime} 11$ & $0^{\prime \prime} 13$ & $1^{\prime \prime} .12$ & \\
\hline 40 & 1994 & Whipple & P0 & $2 / 2$ & $-0^{\prime \prime} 15$ & $0^{\prime \prime} 72$ & $0^{\prime \prime} .53$ & 1.07 & $2 / 2$ & $-0^{\prime \prime} \cdot 40$ & $0 ! 34$ & $0 ! 47$ & 0.93 \\
\hline 41 & 1996 & Whipple & $\mathrm{C} 0$ & $21 / 21$ & $-0^{\prime} ! 37$ & $0 ! 21$ & $0 ! 42$ & 1.06 & $21 / 21$ & $0 ! 11$ & $0 ! 29$ & $0 ! 30$ & 0.75 \\
\hline
\end{tabular}

root-mean-square (wrms), i.e., the rms of the residuals multiplied by their weights. The wrms measures the quality of the fit relative to the assumed accuracy of the observations. The weights can be recovered from the ratio of the wrms to the rms.

Overall, the orbit fits the Earthbased observations at the $1^{\prime \prime}$.19 level. The rms for the "old" (pre-1940) sets is 1".48 and for the "modern" (post-1940) sets is 0 ".78. Among the sets used, the poorest fit is to Barnard's 1906 micrometer measures, and the best fits are to Whipple's 1992 photographic data, Whipple's 1996 CCD data, and Mulholland's 1975 relative photographic data. Also, Nicholson's isolated 1940 observation has a surpris- ingly small residual. Figure 1 displays the right ascension residuals for the observations that were fit, and Fig. 2 displays the declination residuals. The figures give an indication of the time distribution of the observations and the overall quality of the fit. Considerable scatter is evident as well as some suggestion of systematic errors.

The residuals for the Voyager imaging data range in magnitude from 7 to $235 \mathrm{~km}$; the rms for all eight is $104 \mathrm{~km}$.

The rms for residuals of the Saturn observations from Greenwich are 0 ".42 and 0 ".28 in right ascension and declination, respectively. Table 4 gives the biases found for each observation set. 


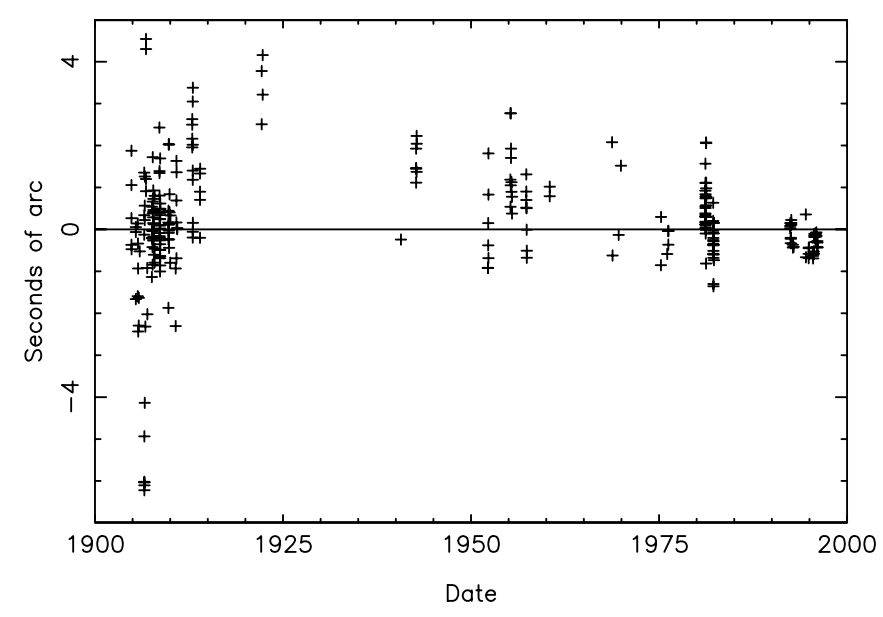

Fig. 1. Right ascension residuals

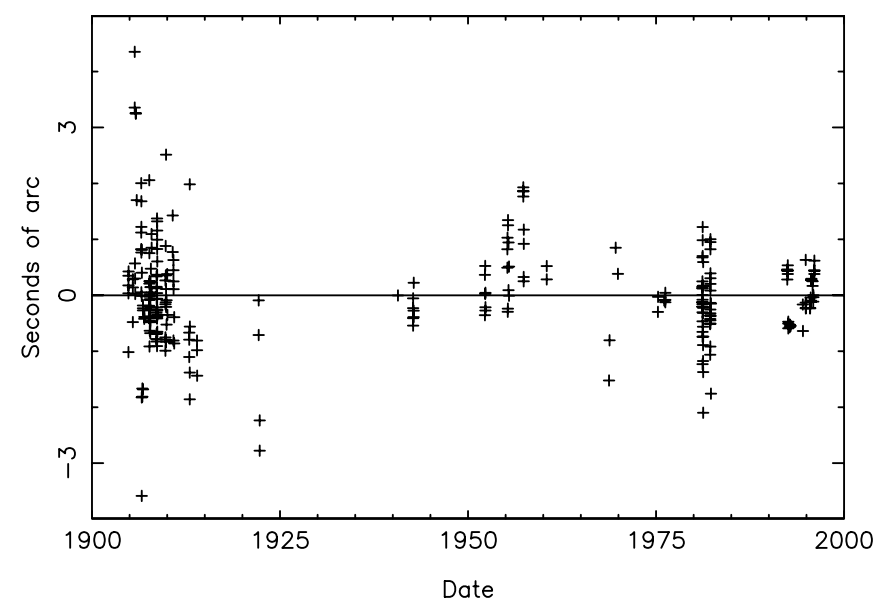

Fig. 2. Declination residuals

\subsection{Epoch state vector}

The epoch state vector obtained from the fit appears in Table 5, and the Saturnian system dynamical constants needed for the integration appear in Table 6. Two state vectors are provided: the first is a from a fit which used the complete dynamical model in post-1966 integration; the second is from a fit which used the simplified model. The simplified model was used in the pre-1966 integration in both cases. The root-mean-square of the differences between the two models over the time period 1900 to 2013 are $7.4 \mathrm{~km}$ in the radial direction, $50.5 \mathrm{~km}$ in the in-orbit direction, and $17.2 \mathrm{~km}$ in the out-of-plane direction.

As an aid to those wishing to reproduce the integration, Table 7 contains the state vector for the simplified model at the end of the integration.
Table 4. Corrections to the mean equator of epoch Greenwich observations

\begin{tabular}{ccr}
\hline Year & Rt. Asc. & \multicolumn{1}{c}{ Dec. } \\
\hline 1907 & $2^{\prime \prime} .88$ & $0^{\prime \prime} .56$ \\
1908 & $1^{\prime \prime} .89$ & $0^{\prime \prime} .57$ \\
1909 & $1^{\prime \prime} 62$ & $-0^{\prime \prime} .20$ \\
1910 & $0^{\prime \prime} .78$ & $0^{\prime \prime} .28$ \\
\hline
\end{tabular}

Table 5. Barycentric state vector at Julian ephemeris date 2439440.50 (1966 Nov. 11.0) referred to the Earth mean equator and equinox of J2000

\begin{tabular}{crr}
\hline Component & Position $(\mathrm{km})$ & \multicolumn{1}{c}{ Velocity $(\mathrm{km} / \mathrm{s})$} \\
\hline \multicolumn{3}{c}{ complete model } \\
\hline$x$ & -12049661.9430915500 & -0.5851428400610138 \\
$y$ & -2354538.7543550990 & 1.5137695265625980 \\
$z$ & 298437.1979645121 & 0.7872087168834739 \\
\hline \multicolumn{3}{c}{ simplified model } \\
\hline$x$ & -12049676.2666544100 & -0.5851329248090125 \\
$y$ & -2354463.3515782810 & 1.5137727228222640 \\
$z$ & 298451.8787930112 & 0.7872099536417393 \\
\hline
\end{tabular}

\subsection{Mean elements}

Because an integrated orbit in terms of cartesian coordinates is difficult to interpret geometrically, an alternative representation in the form of mean orbital elements is often useful. Table 8 provides mean elements derived by fitting a precessing ellipse model to the integration over the period 1900 to 2013. The reference plane is the Phoebe Laplacian plane, the plane on which the orbit precesses almost uniformly. The orientation angles for the Laplacian plane pole are with respect to the Earth mean equator and equinox of J2000 system; the tilt of the plane off the Saturn equator is $26^{\circ} 183$. The epoch mean longitude $\lambda$, longitude of periapsis $\varpi$, and longitude of the ascending node $\Omega$, are measured from the ascending node of the Laplacian plane on the Earth mean equator of J2000. The elements may be used as replacements for those provided by Rohde \& Sinclair (1992) for computing an approximate orbit. We should comment that the latter element set is referred to the ecliptic and equinox of 1950.0, hence the angular elements cannot be compared directly with ours. The root-mean-square of the differences between the integrated and the approximate orbits over the 1900 to 2013 period are $127236 \mathrm{~km}$ in the radial direction, $244253 \mathrm{~km}$ in the in-orbit direction, and $15438 \mathrm{~km}$ in the out-of-plane direction. These differences give an indication of the magnitude of the periodic perturbations (mostly due to the Sun) affecting the orbit. 
Table 6. Saturnian system dynamical constants. These are the constants currently being used in the major Saturnian satellite ephemerides, see Jacobson (1996b)

\begin{tabular}{lrl}
\hline Name & \multicolumn{1}{c}{ Value } & Units \\
\hline Saturn system GM & 37940629.764 & $\mathrm{~km}^{3} / \mathrm{s}^{2}$ \\
Mimas GM & 2.500 & $\mathrm{~km}^{3} / \mathrm{s}^{2}$ \\
Enceladus GM & 4.900 & $\mathrm{~km}^{3} / \mathrm{s}^{2}$ \\
Tethys GM & 41.808 & $\mathrm{~km}^{3} / \mathrm{s}^{2}$ \\
Dione GM & 73.156 & $\mathrm{~km}^{3} / \mathrm{s}^{2}$ \\
Rhea GM & 154.000 & $\mathrm{~km}^{3} / \mathrm{s}^{2}$ \\
Titan GM & 8978.200 & $\mathrm{~km}^{3} / \mathrm{s}^{2}$ \\
Iapetus GM & 106.000 & $\mathrm{~km}^{3} / \mathrm{s}^{2}$ \\
Saturn radius & 60330.0 & $\mathrm{~km}^{2}$ \\
Saturn J & $162.9810^{-4}$ & \\
Saturn J & $213.7410^{-4}$ & \\
Saturn J & $-9.1510^{-4}$ & \\
Saturn J & $1.0310^{-4}$ & \\
Saturn pole right ascension & 40.58 & $\mathrm{deg}$ \\
Saturn pole declination & 83.54 & $\mathrm{deg}$ \\
\hline
\end{tabular}

${ }^{\dagger}$ quadrupole for simplified model.

Table 7. Barycentric state vector for the simplified model at Julian ephemeris date 2414640.5 (1898 Dec. 17.0) referred to the Earth mean equator and equinox of J2000

\begin{tabular}{crr}
\hline Component & \multicolumn{1}{c}{ Position $(\mathrm{km})$} & \multicolumn{1}{c}{ Velocity $(\mathrm{km} / \mathrm{s})$} \\
\hline$x$ & -10039870.733667480 & -1.2513317541446060 \\
$y$ & -6590801.243860413 & 1.1142229491687150 \\
$z$ & -2664829.368414232 & 0.6536617058933396 \\
\hline
\end{tabular}

Table 8. Planetocentric mean orbital elements at Julian ephemeris date 2447892.5 referred to Phoebe's Laplacian plane

\begin{tabular}{lrl}
\hline Element & \multicolumn{1}{l}{ Value } & Units \\
\hline semi-major axis & 12944346 & $\mathrm{~km}$ \\
eccentricity & 0.16435 & \\
inclination & 174.751 & $\mathrm{deg}$ \\
$\lambda$ & 390.742 & $\mathrm{deg}$ \\
$\varpi$ & 203.958 & $\mathrm{deg}$ \\
$\Omega$ & 233.037 & $\mathrm{deg}$ \\
orbital period & 548.2122790 & $\mathrm{days}$ \\
$\mathrm{d} \varpi / \mathrm{d} t$ & 1.19141 & $\mathrm{deg} / \mathrm{yr}$ \\
$\mathrm{d} \Omega / \mathrm{d} t$ & 0.45631 & $\mathrm{deg} / \mathrm{yr}$ \\
Laplacian plane pole & 275.631 & $\mathrm{deg}$ \\
right ascension & & \\
Laplacian plane pole & 68.031 & $\mathrm{deg}$ \\
$\quad$ declination & & \\
\hline
\end{tabular}

\section{Accuracy assessment}

The accuracy to which Phoebe's orbit can be determined is limited primarily by the errors in the observations and by the errors in the ephemeris of Saturn as it affects the modelling of the observations. The DE403 Saturn position error is about $0^{\prime \prime} .2$; the accuracy of computed absolute positions of Phoebe is limited to that value. The majority of the observations are absolute positions obtained from reductions involving a variety of star catalogues and are subject to relatively large systematic errors due to errors in those catalogues (e.g. zone biases, proper motion). Fundamentally, the errors make it difficult to tie the reference frame of the observation to the IERS/J2000 reference frame of the orbit. Characterizing the uncertainty in the frame-tie is extremely difficult, especially for the older observations. Examination of the residuals suggests an overall accuracy of the observed absolute positions in the range from about $0^{\prime \prime} .4$ to $4^{\prime \prime} .0$.

Relative to the observation related errors, those in the dynamical modelling are small. The most important dynamical parameters, the GM's of Saturn and Titan, are well known from the Voyager encounters. Inaccuracies in the ephemerides of the perturbing bodies lead to integration errors of at most a few tens of kilometers.

The effects of any systematic observation errors are alleviated somewhat by the orbit model. The only free dynamical parameters in the orbit determination process are the components of the epoch state vector of Phoebe. Consequently, there exist implicit dynamical constraints on the size, shape, and orientation of possible orbits. It is unlikely, assuming the observations are weighted properly, that the orbit has been distorted in an attempt to accommodate a systematic error unique to a particular observation set.

To arrive at a probable accuracy for the orbit, we first examined the formal covariance from the fit. In the development of that covariance we included ephemeris parameters for Saturn as consider parameters (parameters not estimated but whose uncertainties affect the statistics of the estimated parameters). The uncertainties in the Saturn ephemeris parameters were set to reflect the $0 \prime 2$ error in Saturn's position. The orbit accuracy predicted by the covariance is a lower bound because it only accounts for observation errors as represented by the data weights and for the Saturn ephemeris error. We next made comparisons with fits to various subsets of the data and with fits using differing weighting strategies. Finally, we examined sensitivities to several of the dynamical model parameter values. Based on this analysis, our estimate of the $1 \sigma$ orbit uncertainties at the time of the planned Cassini flyby (June 12, 2004) are:

\begin{tabular}{cccc}
\hline In-orbit & Radial & Out-of-plane & Period \\
\hline $3000 \mathrm{~km}$ & $1000 \mathrm{~km}$ & $1000 \mathrm{~km}$ & $36 \mathrm{~s}$ \\
\hline
\end{tabular}


The period error translates into a growth in the uncertainty of in-orbit direction of roughly $40 \mathrm{~km} / \mathrm{yr}$.

\section{Comparison with previous investigations}

We attempted to repeat the integrations of the other investigators based on the information provided in their publications but were successful only with Rose's orbit (the measure of success was our ability to closely reproduce the published residual statistics). Failure in the other two cases is most likely due to the inability of our software to duplicate the perturbations included by the other investigators. One problem with the Bec-Borsenberger and Rocher integration is that they did not give the value of Titan's mass used in their Titan perturbation.

We were able to match Rose's orbit because he provided state vectors at both the beginning and end of his integration; we adjusted his initial velocities slightly to ensure that our integration matched his initial and final positions. The integration was performed in the FK4/B1950 system with solar perturbations computed using JPL planetary ephemeris DE102 (Newhall et al. 1995). Rose fit his orbit to 133 observations which he had converted to the FK4/B1950 system. He included the converted observations in his paper but did not provide details of his conversion procedure. He found a standard error of $1^{\prime \prime} .52$; our reproduction of his orbit gives a standard error of 1 ".46 for those published FK4/B1950 observations. For the pre1970 observations that we used our work, the respective standard errors for our orbit and Rose's orbit are 1".40 and $1^{\prime \prime} .78$; for the post- 1970 observations, they are $0^{\prime \prime} .60$ and $1^{\prime \prime} 32$. The root-mean-square of the differences between our orbit and that of Rose over the time period 1966-2001 are $4248 \mathrm{~km}$ in the radial direction, $10121 \mathrm{~km}$ in the in-orbit direction, and $3405 \mathrm{~km}$ in the out-of-plane direction.

Except for the Arequipa data, Bykova and Shikhalev and Bec-Borsenberger and Rocher fit essentially the same observations. Like Rose, each pair of investigators converted the observations to the FK4/B1950 system before processing. Bykova and Shikhalev give a standard error of $1^{\prime \prime} .5$ (with some unspecified subset of the Arequipa data included), and Bec-Borsenberger and Rocher quote $\Delta \alpha \cos \delta$ and $\Delta \delta$ errors of $1^{\prime \prime} .7$ and $1^{\prime \prime} .2$, respectively. For the pre1982 observations used our work, our orbit yields respective errors $\Delta \alpha \cos \delta$ and $\Delta \delta$ of $1^{\prime \prime} .56$ and $1^{\prime \prime} .00$.

Bec-Borsenberger and Rocher give sets of polynomial coefficients from which geocentric astrometric positions can be computed for the years 1981-1990 based on their orbit. From those polynomials we constructed positions for the first of January of each of those years and compared them to computed positions from our orbit. In the comparison we assumed that the constructed positions were in the reference frame of DE102, the planetary ephemeris used in the analysis, and transformed them with the published rotation between the J2000 frame and that of DE102. The rms of $\Delta \alpha \cos \delta$ and $\Delta \delta$ residuals are $0^{\prime \prime} .26$ and $0^{\prime \prime} .30$.
It appears that our new orbit agrees with that of BecBorsenberger and Rocher at a level better than our current orbit accuracy.

\section{Conclusions}

This article has reported on the determination of the orbit of Phoebe using a numerical integration fit to Earthbased astrometric observations and imaging observations obtained from the Voyager 2 spacecraft. It has included a description of the dynamical models used in the integration, a general discussion of the observations available, an overview of the observation processing procedure, an assessment of the accuracy of the final orbit, and a comparison of the orbit with previously published integrations.

Because of its distance from Saturn, astrometric measures of Phoebe are mostly and will probably continue to be absolute positions. Any future determination of an improved orbit incorporating such positions will require both high quality observations and careful reduction with new star catalogues closely tied to the IERS/J2000 reference frame. Re-reduction of the existing observations against such catalogues would also provide significant benefit.

The determination of the orbits of most other planetary satellites relies heavily on intersatellite and planet relative positions, observations which are independent of star catalogue related errors. Among the existing Phoebe observations, there are a number of relative positions, but most are no better than the best absolute positions. New precise wide field simultaneous observations of Phoebe together with Saturn or other Saturnian satellites could provide invaluable relative positions leading to a greatly improved orbit.

An ephemeris based on the orbit described in this article is available electronically from the JPL Horizons online solar system data and ephemeris computation service (Giorgini et al. 1996).

Acknowledgements. The research described in this publication was carried out by the Jet Propulsion Laboratory, California Institute of Technology, under a contract with the National Aeronautics and Space Adminstration. The author wishes to thank all of the astronomers who have provided the astrometric observations of Phoebe. The author also wishes to thank J.H. Lieske for his assistance in interpreting many of the old publications.

\section{Appendix A: Approximate Titan orbit}

The orbit of Titan used in computing the perturbations in the simplified model is defined by a set of orbital elements which were derived from a fit to the SAT077 integrated Titan orbit. Because of Titan's small eccentricity and inclination, we adopted the equinoctial form of the elements (Broucke \& Cefola 1972); Table 9 contains the elements. The table also gives the orientation angles for Titan's Laplacian plane in the Earth mean 
equator and equinox of J2000 system. The orbital longitudes $(\lambda, \varpi, \Omega)$ are measured from the ascending node of the Laplacian plane on the Earth mean equator of J2000. The root-mean-square of the differences between the Titan orbit defined by the elements and the integrated orbit are $72 \mathrm{~km}$ in the radial direction, $264 \mathrm{~km}$ in the in-orbit direction, and $210 \mathrm{~km}$ in the out-of-plane direction over the 1966 to 2013 time period.

When computing the perturbations on Phoebe, the Titan position relative to the Saturnian system barycenter is found with the elements, and then the position of Saturn relative to the barycenter is formed from the Titan position assuming that Titan and Saturn are the only massive bodies in the Saturnian system.

Table 9. Titan barycentric orbital elements at Julian ephemeris date 2451179.5 referred to Titan's Laplacian plane

\begin{tabular}{lll}
\hline Element & \multicolumn{1}{c}{ Value } & Units \\
\hline$a$ & 1221577. & $\mathrm{~km}$ \\
$h=e \sin \varpi$ & $-1.1484710^{-2}$ & \\
$k=e \cos \varpi$ & $-2.6361310^{-2}$ & \\
$\lambda$ & 395.675178 & $\mathrm{deg}$ \\
$p=\tan (I / 2) \sin \Omega$ & $-0.2822010^{-2}$ & \\
$q=\tan (I / 2) \cos \Omega$ & $-0.4100810^{-2}$ & \\
$\mathrm{~d} \lambda / \mathrm{d} t$ & $2.6130757958010^{-4}$ & $\mathrm{deg} / \mathrm{s}$ \\
$\mathrm{d} \varpi / \mathrm{d} t$ & $1.633780010^{-8}$ & $\mathrm{deg} / \mathrm{s}$ \\
$\mathrm{d} \Omega / \mathrm{d} t$ & $0.938456010^{-8}$ & $\mathrm{deg} / \mathrm{s}$ \\
Laplacian plane pole & 40.581056 & $\mathrm{deg}$ \\
right ascension & & \\
Laplacian plane pole & 83.225166 & $\mathrm{deg}$ \\
declination & & \\
\hline
\end{tabular}

\section{Appendix B: Voyager observations and processing}

The Voyager observations of Phoebe appear in Table 10 in the form of the pixel and line locations in the Voyager camera frame. The table also gives the inertial camera pointing angles which were derived from the background star images. As a simplification, the effects of uncertainty in the pointing have been incorporated into the location accuracies quoted in the table. The observations have not been corrected for stellar aberration; they provide measures of the apparent position of Phoebe as seen from the spacecraft.

To process the observations, first compute the apparent position of Phoebe:

$$
\begin{aligned}
\boldsymbol{A}= & {[\boldsymbol{s}(t-\tau)+\boldsymbol{b}(t-\tau)]-[\boldsymbol{r}(t)+\boldsymbol{b}(t)] } \\
& +\tau[\dot{\boldsymbol{r}}(\mathbf{t})+\dot{\boldsymbol{b}}(\mathbf{t})]
\end{aligned}
$$

where $t$ is the observation time (ET), $\tau$ is the light travel time from the Phoebe to the spacecraft, $s$ is Phoebe's po- sition vector, $\boldsymbol{r}$ is the spacecraft position vector, and $\boldsymbol{b}$ is the Saturnian system barycenter position vector; the former two positions are relative to the Saturnian system barycenter, and the latter is relative to the Solar System barycenter. The vectors $\dot{\boldsymbol{r}}$ and $\boldsymbol{b}$ are the respective velocities of the spacecraft and Saturnian system barycenter. This computation implicitly involves an iterative procedure to determine $\tau$ from the true relative distance between Phoebe and the spacecraft. Next rotate the apparent position into camera body coordinates:

$\boldsymbol{P}=\boldsymbol{R}_{3}(\phi) \boldsymbol{R}_{2}\left(90^{\circ}-\delta\right) \boldsymbol{R}_{3}(\alpha) \boldsymbol{A}$

where $\alpha, \delta, \phi$ are the right ascension, declination, and twist camera pointing angles from Table 10, and $\boldsymbol{R}_{i}$ is the standard $3 \times 3$ rotation matrix about the $i_{\text {th }}$ axis. Then project the camera body coordinates into the focal plane via the gnomonic projection:

$\left(\begin{array}{l}x \\ y\end{array}\right)=\frac{f}{P_{3}}\left(\begin{array}{l}P_{1} \\ P_{2}\end{array}\right)$

where $f$ is the focal length of the camera and $P_{i}$ is the $i_{\text {th }}$ component of the vector $\boldsymbol{P}$. The focal plane coordinates must be corrected for electromagnetic and optical distortion:

$\left(\begin{array}{l}x^{\prime} \\ y^{\prime}\end{array}\right)=\left(\begin{array}{l}x \\ y\end{array}\right)+\left(\begin{array}{rrrrrr}-y r & x r^{2} & -y r^{3} & x r^{4} & x y & x^{2} \\ x r & y r^{2} & x r^{3} & y r^{4} & y^{2} & x y\end{array}\right)\left(\begin{array}{l}e_{1} \\ e_{2} \\ e_{3} \\ e_{4} \\ e_{5} \\ e_{6}\end{array}\right)$

where $r^{2}=x^{2}+y^{2}$ and the $e_{i}$ are the distortion coefficients. Lastly convert from the focal plane coordinates into pixel and line:

$$
\left(\begin{array}{l}
p \\
l
\end{array}\right)=\left(\begin{array}{lll}
K_{x} & K_{x y} & K_{x x y} \\
K_{y x} & K_{y} & K_{y x y}
\end{array}\right)\left(\begin{array}{c}
x^{\prime} \\
y^{\prime} \\
x^{\prime} y^{\prime}
\end{array}\right)+\left(\begin{array}{c}
p_{0} \\
l_{0}
\end{array}\right)
$$

where $\boldsymbol{K}$ is the camera transformation matrix and $p_{0}, l_{0}$ are the pixel and line location of the optical axis.

For the Voyager 2 narrow angle camera the focal length is $1503.49 \mathrm{~mm}$, the optical axis is located at (398.07, $401.95)$, the elements of the conversion matrix $\boldsymbol{K}$ are:

$$
\left(\begin{array}{lll}
K_{x} & K_{x y} & K_{x x y} \\
K_{y x} & K_{y} & K_{y x y}
\end{array}\right)=\left(\begin{array}{rrr}
72.5270 & 0.5619 & 0.001433 \\
-0.7227 & 72.9500 & -0.008309
\end{array}\right)
$$

and the distortion coefficients are:

$$
\begin{aligned}
e= & \left(-1.11510^{-4}, 1.16910^{-4},-2.47510^{-5},\right. \\
& \left.-1.00810^{-5},-2.33510^{-4},-2.11010^{-4}\right)
\end{aligned}
$$

Table 11 contains the Voyager position, $\boldsymbol{r}$, and velocity, $\dot{\boldsymbol{r}}$, vector components at the observation times. 
Table 10. Voyager 2 imaging observations. The observation time is the midtime of the exposure. The pointing angles are in the Earth mean equator and equinox of J2000 system. The observation accuracies, denoted by Acc., include the effects of the camera pointing uncertainty

\begin{tabular}{lcccccccc}
\hline Picture ID & Observation time (UTC) & \multicolumn{3}{c}{ Camera pointing angles (deg) } & \multicolumn{2}{c}{ Pixel } & \multicolumn{2}{c}{ Line } \\
& & Rt. Ascen. & Dec. & Twist & Value & Acc. & Value & Acc. \\
\hline 41901B+37 & 17 Jun. 1981 00:11:52.12 & 203.323974 & -7.744627 & -129.273157 & 465.80 & 0.71 & 589.34 & 0.61 \\
42182B+27 & 26 Jun. 1981 08:51:52.12 & 205.345157 & -8.780149 & -127.860860 & 492.73 & 0.60 & 402.65 & 0.58 \\
$42351 B+55$ & 02 Jul. 1981 00:26:11.21 & 206.845295 & -9.510055 & -127.253253 & 571.33 & 0.67 & 319.07 & 0.65 \\
$42800 B+50$ & 16 Jul. 1981 23:34:11.21 & 212.089857 & -11.710938 & -123.628497 & 372.56 & 0.58 & 381.50 & 0.58 \\
$43300 B+39$ & 02 Aug. 1981 15:25:32.81 & 222.738376 & -15.963989 & -118.780588 & 422.66 & 0.70 & 140.20 & 0.58 \\
$43461 B+08$ & 07 Aug. 1981 23:48:44.81 & 228.634792 & -17.725684 & -115.402728 & 339.80 & 0.70 & 547.97 & 0.62 \\
$43491 B+27$ & 09 Aug. 1981 00:03:56.81 & 230.085968 & -18.288527 & -115.301906 & 642.37 & 0.57 & 388.94 & 0.58 \\
$43696 B+50$ & 15 Aug. 1981 20:22:19.85 & 241.941386 & -21.485373 & -110.265804 & 323.33 & 0.66 & 399.83 & 0.66 \\
\hline
\end{tabular}

Table 11. Voyager 2 Saturn barycentric position and velocity referred to the Earth mean equator and equinox of J2000

\begin{tabular}{lrrr}
\hline Picture ID & \multicolumn{3}{c}{ Position $(\mathrm{km})$ and Velocity $(\mathrm{km} / \mathrm{s})$} \\
& \multicolumn{1}{c}{$x$} & \multicolumn{1}{c}{$y$} & \multicolumn{1}{c}{$z$} \\
\hline $41901 \mathrm{~B}+37$ & 63212533.0 & 19188537.5 & 4918608.4 \\
& -10.271438 & -3.060466 & -0.771611 \\
$42182 \mathrm{~B}+27$ & 54904032.8 & 16712535.5 & 4294300.1 \\
& -10.274296 & -3.062307 & -0.772204 \\
$42351 \mathrm{~B}+55$ & 49888808.0 & 15217592.1 & 3917311.5 \\
& -10.277604 & -3.063816 & -0.772655 \\
$42800 \mathrm{~B}+50$ & 36591667.8 & 11252598.7 & 2917747.4 \\
& -10.295356 & -3.070303 & -0.774463 \\
$43300 \mathrm{~B}+39$ & 21738982.8 & 6823497.4 & 1799317.7 \\
& -10.350959 & -3.087303 & -0.779872 \\
$43461 \mathrm{~B}+08$ & 16946391.4 & 5393865.6 & 1438146.6 \\
& -10.390964 & -3.100103 & -0.783285 \\
$43491 \mathrm{~B}+27$ & 16038694.3 & 5123047.7 & 1369718.5 \\
& -10.401263 & -3.103412 & -0.784171 \\
$43696 \mathrm{~B}+50$ & 9856547.4 & 3277698.5 & 903262.8 \\
& -10.521137 & -3.142626 & -0.794836 \\
\hline
\end{tabular}

\section{References}

Albrecht S., Smith E., 1909, Lick Obs. Bull. 156, 109

Astronomical Almanac, 1987, U.S. Government Printing Office, Washington DC, B36

Barnard E.E., 1905, AJ 24, 165

Barnard E.E., 1906, Astron. Nachr. 177, 4234, 10

Barnard E.E., 1913, Astron. Nachr. 194, 4651, 333

Barnard E.E., 1914, Astron. Nachr. 198, 4740, 223

Bec-Borsenberger A., Rocher P., 1982, A\&AS 50, 423

Bobone J., 1953, AJ 58, 172

Bowell E., 1981, IAU Circ. No. 3602

Bowell E., 1982, IAU Circ. No. 3719

Bowell E., 1988 (private communication of Lowell Observatory astrometric observations)

Broucke R.A., Cefola P.J., 1972, Celest. Mech. 5, 303
Bykova L.E., Shikhalev V.V., 1982, Astron. Tsirk. 1237

Bykova L.E., Shikhalev V.V., 1984, Celest. Mech. 32, 185

Chernykh L.I., Chernykh N.S., 1971, Bul. Inst. Th. Astron. XII, 8 (141)

Christie W.H.M., 1909, Greenwich Obs. 1907197

Christie W.H.M., 1910, Greenwich Obs. 1908, 181

Christie W.H.M., 1911, Greenwich Obs. 1909, 159

Christie W.H.M., 1912, Greenwich Obs. 1910, C119

Debehogne H., 1981a, IAU Circ. No. 3612

Debehogne H., 1981b, IAU Circ. No. 3629

Debehogne H., 1981c, IAU Circ. No. 3654

Debehogne H., 1981d, IAU Circ. No. 3707

Debehogne H., 1982 (private communication to A. BecBorsenberger, appears in Bec-Borsenberger A., Rocher P., 1982, A\&AS 50, 423)

Debehogne H., 1984, Bull. Astron. 9, 299

Explanatory Supplement to the Astronomical Ephemeris and the American Ephemeris and Nautical Almanac, 1961, Her Majesty's Stationary Office, London

Giorgini J.D., Yeomans D.K., Chamberlin A.B., 1996, 28th Annual Meeting of the Division for Planetary Sciences of the American Astronomical Society, Tucson AZ

Jacobson R.A., 1996a, "Update of the Major Saturnian Satellite Ephemerides" IOM 312.1-96-012, Jet Propulsion Laboratory, Pasadena, CA (internal document)

Jacobson R.A., 1996b, 1996 Meeting of the American Astronomical Society Division on Dynamical Astronomy, US Naval Observatory, Washington DC

Klemola A.R., Taraji H., Ocampo A., 1979, Lick SaturnVoyager Reference Star Catalogue Lick Observatory, Santa Cruz, California

Kohlhase C., 1993, The Planetary Report, 13, 5

Lawson C.L., Hanson R.J., 1974, Solving Least Squares Problems, Prentice-Hall, Englewood Cliffs N.J.

Mulholland J.D., Shelus P.J., 1980, AJ 85, 1112

Murray C.A., 1983, Vectorial Astrometry. Adam Hilger Ltd, Bristol

Nicholson S.B., Richmond M., 1944, AJ 50, 163

Newhall X.X., Standish E.M., Williams J.G., 1983, A\&A 125, 150

Owen W.M., Vaughan R.M., 1991, "Optical Navigation Program User's Guide", Jet Propulsion Laboratory 
Engineering Memorandum 314-512, Jet Propulsion Laboratory, Pasadena, CA (internal document)

Paris conference, 1911, Congrés International des Ephémérides Astronomiques tenu à l'Observatoire de Paris, 23-26 Octobre 1911

Pierce D.A., 1975, JPL Tech. Memo. 900-698, Jet Propulsion Laboratory Pasadena, CA (internal document)

Pickering E.C., 1899, Harv. Coll. Obs. Circ. 43

Pickering E.C., 1904, Harv. Coll. Obs. Circ. 87

Pickering E.C., 1906a, Harv. Coll. Obs. Circ. 109

Pickering E.C., 1906b, Harv. Coll. Obs. Circ. 118

Pickering E.C., 1906c, Harv. Coll. Obs. Circ. 119

Pickering W.H., 1905a, Ann. Harv. Coll. Obs. LIII, III, 45

Pickering W.H., 1905b, Ann. Harv. Coll. Obs. LIII, V, 85

Pickering W.H., 1908, Ann. Harv. Coll. Obs. LX, III, 45

Perrine C.D., 1904, Lick Obs. Bull. 64, 52

Perrine C.D., 1909, Lick Obs. Bull. 156, 106

Peters C.F., 1981, A\&A 104, 37

Roemer E., Lloyd R.E., 1966, AJ 71, 443

Rohde J.R., Sinclair A., 1992, in Explanatory Supplement to the Astronomical Almanac Seidelmann P.K., (ed). University Science Books, Mill Valley, CA

Rohde J.R., 1994 (private communication of USNO astrometric observations) 84

Rose L.E., 1979, AJ 1067
Ross F.E., 1905, Ann. Harv. Coll. Obs. LIII VI, 101

Roy A.E., Walker I.W., Macdonald A.J., Carpino M., 1988, Vistas Astron. 32, 95

Standish E.M., Newhall X.X., Williams J.G., Yeomans D.K., 1992, in Explanatory Supplement to the Astronomical Almanac Seidelmann P.K., (ed). University Science Books, Mill Valley, CA

Standish E.M., Newhall X.X., Williams J.G., Folkner W.M., 1995, "JPL Planetary and Lunar Ephemerides DE403/ LE403", IOM 314.10-127, Jet Propulsion Laboratory Pasadena, CA (internal document)

van Biesbroeck G., 1922, AJ 34, 167

van Biesbroeck G., 1944, AJ 50, 184

van Biesbroeck G., 1956, AJ 62, 136

van Biesbroeck G., 1958, AJ 63, 147

van Biesbroeck G., Vesely C.D., Aksnes K., Marsden B.G., 1976, AJ 81, 122

Whipple A.L., 1992 (private communication of McDonald Observatory astrometric observations)

Whipple A.L., 1995 (private communication of McDonald Observatory astrometric observations)

Whipple A.L., 1996 (private communication of McDonald Observatory astrometric observations)

Zadunaisky P.E., 1954, AJ 59, 1 